\title{
Molecular determinants of short chain fatty acid production: influence of glycosidic bond configuration
}

\author{
H. C. Harris ${ }^{1,2}$, C. A. Edwards ${ }^{1}$ and D. J. Morrison ${ }^{2}$ \\ ${ }^{1}$ Human Nutrition, School of Medicine, University of Glasgow, New Lister Building, Glasgow, United Kingdom, \\ G31 2ER and ${ }^{2}$ SUERC, University of Glasgow, East Kilbride, United Kingdom, G75 0QF
}

Short chain fatty acids (SCFA) are produced by the fermentation of dietary fibre in the colon. The main SCFA produced are acetate, propionate, and butyrate and each may have a specific and separate role in health. For example, propionate may have satiating effects while butyrate may have a role in the prevention of cancer. There are a variety of factors that determine the rate and extent of SCFA production; one of these is glycosidic bond configuration in polysaccharides. For example, $\beta(1-4)$ and $\beta(1-3)$ bonding in $\beta$-glucan results in increased propionate production ${ }^{(1)}$ and $\alpha(1-4)$ and $\alpha(1-6)$ bonding in starch results in increased butyrate production ${ }^{(2)}$. In this study, we looked at the effect of bonding structure in model glucose disaccharides on SCFA production. We carried out in vitro fermentations with a miniaturised batch culture technique $(n=9)$, with all commercially available diglucose stereoisomers: Trehalose $\alpha(1-1) \alpha$, Kojibiose $\alpha(1-2)$, Nigerose $\alpha(1-3)$, Maltose $\alpha(1-4)$, Isomaltose $\alpha(1-6)$, Trehalose $\alpha(1-1) \beta$, Sophorose $\beta(1-2)$, Laminaribiose $\beta(1-3)$, Cellobiose $\beta(1-4)$, Gentiobiose $\beta(1-6)$. A faecal blank was used as control. SCFA production was analysed using GC-FID with 2-ethyl butyrate as internal standard. Statistical analysis was carried out by ANOVA with post-hoc Bonferonni correction.

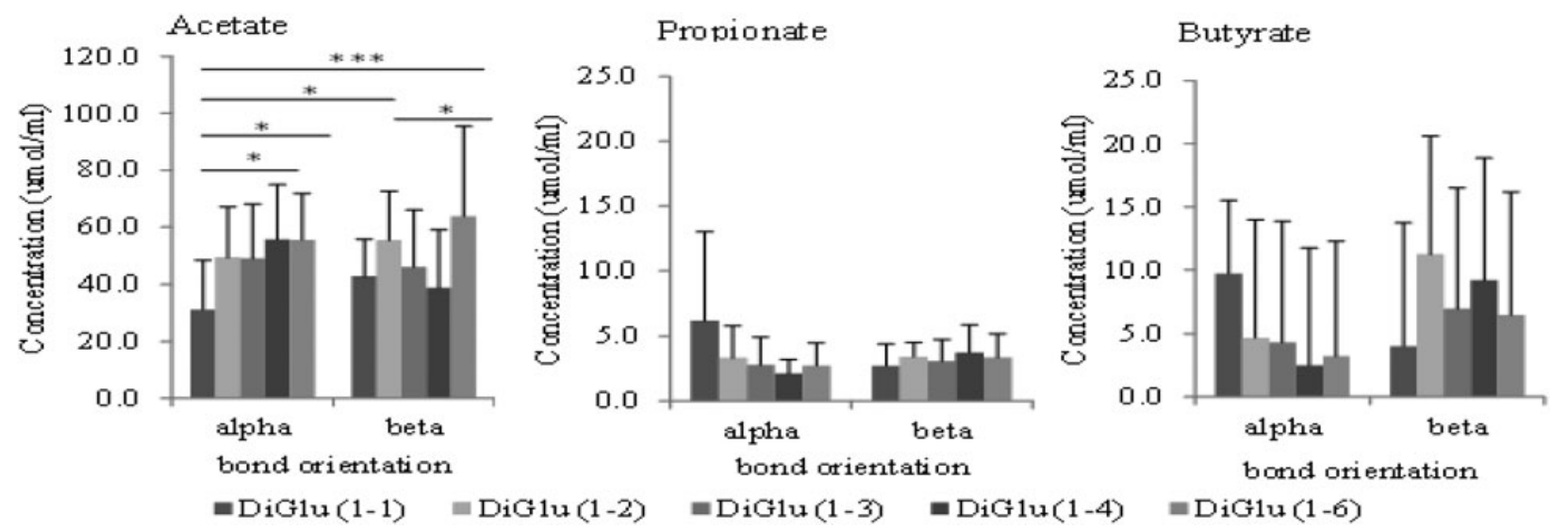

Fig. 1: SCFA concentrations in culture supernatants after 24 hours anaerobic incubation (mean $+/-$ SD), $n=9$ (except beta Digluc $(1-6)$ and $(1-1)(n=$ 6 and $n=8$ respectively). NB alpha Digluc $(1-1)$ is $\alpha(1-1) \beta .{ }^{*} p<0 \cdot 05,{ }^{* * *} p<0 \cdot 001$. Bars in order of list above.

Trehalose $(\alpha(1-1) \alpha)$ produced significantly lower acetate compared to maltose $(\alpha(1-4))$ and all beta bonds (except Laminaribiose $(\beta(1-3))$, however this substrate is also associated with higher proportions of propionate and butyrate (although this is non-significant). All substrates with $\beta$ bonding resulted in a general increase in butyrate production this was also identified to a lesser extent in propionate. There were large intra-individual differences within the population. These results suggest that glycosidic bond configuration between carbohydrate monomers is not the dominant feature involved in determining the pattern of SCFA production by colonic microbiota. The role of glycoside endo- and exohydrolases that interact with polysaccharide structures merit further investigation to unravel the determinants of SCFA production patterns.

1. Hughes SA, Shewry PR, Gibson GR et al. (2008) FEMS microbial ecol., 64

2. Topping DL and Clifton PM (2001) Physiological Reviews., 81(3). 